\title{
Erratum to: Physiological and transcriptional analysis of the effects of formaldehyde exposure on Arabidopsis thaliana
}

\author{
Sha-Sha Wang $\cdot$ Zhong-Bang Song $\cdot$ Zhen Sun • \\ Jing Zhang $\cdot$ Yan Mei $\cdot$ Hong-Juan Nian • \\ Kun-Zhi Li $\cdot$ Li-Mei Chen
}

Published online: 28 January 2012

(C) Franciszek Górski Institute of Plant Physiology, Polish Academy of Sciences, Kraków 2012

\section{Erratum to: Acta Physiol Plant}

DOI 10.1007/s11738-011-0889-3

The affiliations of the authors were not mentioned precisely in the original publication. The correct affiliations are given below:

Sha-Sha Wang

Faculty of Environmental Science and Engineering, Kunming University of Science and Technology, Chenggong, Kunming 650500, China
Sha-Sha Wang · Zhong-Bang Song · Zhen Sun · Jing Zhang · Yan Mei · Hong-Juan Nian · Kun-Zhi Li · Li-Mei Chen $(\square)$ Faculty of Life Science and Biotechnology, Kunming University of Science and Technology, Chenggong, Kunming 650500, China e-mail: chenlimeikm@126.com

The online version of the original article can be found under doi:10.1007/s11738-011-0889-3.

\section{S.-S. Wang}

Faculty of Environmental Science and Engineering,

Kunming University of Science and Technology,

Chenggong, Kunming 650500, China

S.-S. Wang - Z.-B. Song - Z. Sun - J. Zhang · Y. Mei ·

H.-J. Nian · K.-Z. Li · L.-M. Chen ( $\square)$

Faculty of Life Science and Biotechnology,

Kunming University of Science and Technology,

Chenggong, Kunming 650500, China

e-mail: chenlimeikm@126.com 